\title{
No association of the MTHFR gene A1298C polymorphism with the risk of prostate cancer: A meta-analysis
}

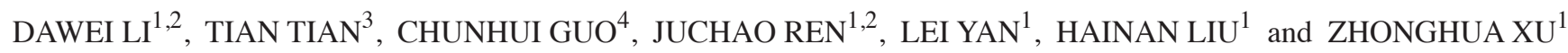 \\ ${ }^{1}$ Department of Urology, Qilu Hospital of Shandong University, Jinan, Shandong 250012; \\ ${ }^{2}$ Key Laboratory of Cardiovascular Remodeling and Function Research Affiliated with the Ministry of Education \\ and the Ministry of Public Health of P.R. China, Jinan, Shandong 250012; Departments of ${ }^{3}$ Tuberculosis \\ and ${ }^{4}$ Oncology, Tuberculosis Hospital of Binzhou City, Binzhou, Shandong 251700, P.R. China
}

Received September 19, 2011; Accepted November 24, 2011

DOI: $10.3892 / \mathrm{etm} .2012 .445$

\begin{abstract}
Various studies have demonstrated that the 5,10-methylenetetrahydrofolate reductase (MTHFR) gene polymorphism contributes to the risk of prostate cancer, while other studies have provided conflicting findings. In the present study, we carried out a comprehensive meta-analysis with the aim of determining whether there is a significant association of the MTHFR gene A1298C polymorphism with the susceptibility of prostate cancer. Studies on the MTHFR gene A1298C polymorphism and prostate cancer were retrieved using the electronic PubMed database without any restriction on language through Aug 21, 2011. Data were abstracted by a standardized protocol. Crude odds ratios (ORs) and 95\% confidence intervals (CIs) were calculated to estimate the strength of association. The analyses were conducted with Review Manager software version 4.2. Nine case-control studies were identified, including 2,723 prostate cancer patients and 3,442 controls. Overall, no significant associations were found between the MTHFR gene A1298C polymorphism and prostate cancer (codominant models: $\mathrm{CC}$ vs. AA, OR=1.03, 95\% CI 0.79-1.34, $\mathrm{P}=0.84$; AC vs. AA, OR=1.04, 95\% CI 0.93-1.16, $\mathrm{P}=0.46$; dominant model: $\mathrm{AC}+\mathrm{CC}$ vs. $\mathrm{AA}, \mathrm{OR}=1.04,95 \% \mathrm{CI}$ 0.94-1.15, $\mathrm{P}=0.48$; recessive model: $\mathrm{CC}$ vs. $\mathrm{AC}+\mathrm{AA}, \mathrm{OR}=1.02$, 95\% CI 0.76-1.35, $\mathrm{P}=0.91$; allele model: $\mathrm{C}$ vs. $\mathrm{A}, \mathrm{OR}=1.04$, $95 \%$ CI $0.90-1.19, \mathrm{P}=0.61)$. Similarly, in the subgroup analyses by DNA source, ethnicity, control source, pathological stage and Hardy-Weinberg equilibrium, no significant associations were observed. Our meta-analysis suggests that the MTHFR gene A1298C polymorphism is not associated with the risk of prostate cancer.
\end{abstract}

Correspondence to: Dr Zhonghua Xu or Dr Lei Yan, Department of Urology, Qilu Hospital of Shandong University, Wenhuaxi Road \#107, Jinan, Shandong 250012, P.R. China

E-mail: xuzhonghuamd@yahoo.com.cn or yanlei5309@sina.com

Key words: 5,10-methylenetetrahydrofolate reductase, polymorphism, meta-analysis, prostate cancer

\section{Introduction}

Prostate cancer (PC) is the most common malignancy and the second leading cause of cancer-related death in men in industrialized countries $(1,2)$. Its incidence is at a relatively low rate in the Asian population (3), but is increasing rapidly (4). It is supposed that complex elements, such as hormones, age, family history of PC, cultural and enviromental factors and genetic background (3), contribute to the cancerization and progression of PC. However, the specific mechanism remains undetermined.

Folate is indispensably required for DNA synthesis and methylation of DNA and histones. Epidemiological studies have shown an effective association between low folate intake and an increased cancer risk $(5,6)$. MTHFR plays a vital role in the metabolism of folates by irreversibly converting 5,10-methylenetetrahydrofolate to 5-methylenetetrahydrofolate (7), which donates a methyl group for the remethylation of homocysteine to methionine used for DNA synthesis and repair (8). Therefore, MTHFR deficiency may lead to DNA hypomethylation to initialize cancerization and affect the progression of malignant tumors $(9,10)$.

The human MTHFR gene, composed of 11 exons, is located at chromosome 1p36.3, codes cDNA of 2.2-kb in length and produces a protein of 656 amino acids (11). The 1298A >C polymorphism, marked as rs1801131 in the NCBI database, is located at exon 7 and results in a glutamate-to-valine substitution at codon 429 (8). Alterations in genomic bases result in single-nucleotide polymorphisms (SNPs), which may subsequently affect the genetic instability, amino acid sequence and function of protein. Recently, SNPs have been used as a tool for predicting diseases $(12)$ in addition to carcinogenesis $(13,14)$. The MTHFR gene A1298C polymorphism has been implicated in several diseases $(15,16)$, including various types of cancer $(17,18)$, and has been investigated in relation to the risk of PC but with inconclusive results (19-27). Among the nine eligible casecontrol studies, three considered the MTHFR gene A1298C polymorphism as a genetic marker for PC $(19,24,26)$, while six reported negative associations between the two $(20-23,25,27)$. Hence, we carried out a meta-analysis concerning the association between the MTHFR gene A1298C polymorphism and PC susceptibility by pooling data from the identified studies to obtain a more conclusive estimation. 


\section{Materials and methods}

Identification of relevant studies. Publications were identified by a systematic electronic search in the PubMed database with the following keywords: 'methylenetetrahydrofolate reductase', 'MTHFR', 'polymorphism', 'variation', 'mutation', 'prostate' and 'prostatic', as well as their combinations. The last search was updated on Aug 21,2011. We did not set any restriction on the language of the published literature. Additional studies were searched by manually screening references in review articles and original papers.

Inclusion and exclusion criteria. The inclusion criteria used for the article selection in this meta-analysis were as follows: i) case-control study with PC and control groups; ii) study focusing on the association between the MTHFR gene A1298C polymorphism and the susceptibility of PC; iii) frequencies of the various genotypes in the publications were available.

The major exclusion criteria were studies that were duplication of a previous publication, studies without detailed information, or not case-control studies, such as review articles, case reports, editorials, conference abstracts and letters.

Data extraction. Two investigators (D.L. and C.G.) reviewed and extracted the information from all included publications independently by a standardized protocol, according to the inclusion and exclusion criterias. Characteristics, such as year of publication, name of first author, country of origin, ethnicity, source of control group, methods for detecting the MTHFR gene A1298C polymorphism, C allele percentage in controls and frequencies of $\mathrm{AA}, \mathrm{AC}$ and $\mathrm{CC}$ genotypes in the case and control groups, were respectively extracted from the included studies. In the case of disagreement, discrepancies of included studies were resolved by discussion.

Statistical analysis. We predicted the contribution of the MTHFR gene A1298C polymorphism to the risk of PC by adopting the Review Manager software version 4.2 developed by the Cochrane Collaboration. The strength of association was estimated by calculating summary crude odds ratios (ORs) and the corresponding $95 \%$ confidence intervals (CIs). We evaluated the risk of the dominant $(\mathrm{CC}+\mathrm{AC}$ vs. $\mathrm{AA})$, recessive (CC vs. $\mathrm{AA}+\mathrm{AC}$ ), allele (C vs. A) and codominant models (AC vs. AA; CC vs. AA), respectively. Hardy-Weinberg equilibrium (HWE) for the control groups in each study was checked by the goodness-of-fit test. Heterogeneity assumption was assesed by the Chi-square-based Q test and was regarded to be statistically significant at $\mathrm{P}<0.10$. The random-effects model was used when the test of heterogeneity was significant, otherwise the fixed-effects model was applied in the analysis. Potential publication bias was primarily appraised by the funnel plot. An asymmetric plot suggested a possible publication bias. Funnel plot asymmetry was further evaluated by Egger's linear regression and Begger's rank correlation tests with STATA software, version 7.0. All P-values were two-tailed.

\section{Results}

Study characteristics. Nine published studies were eligible for this meta-analysis on the association between MTHFR

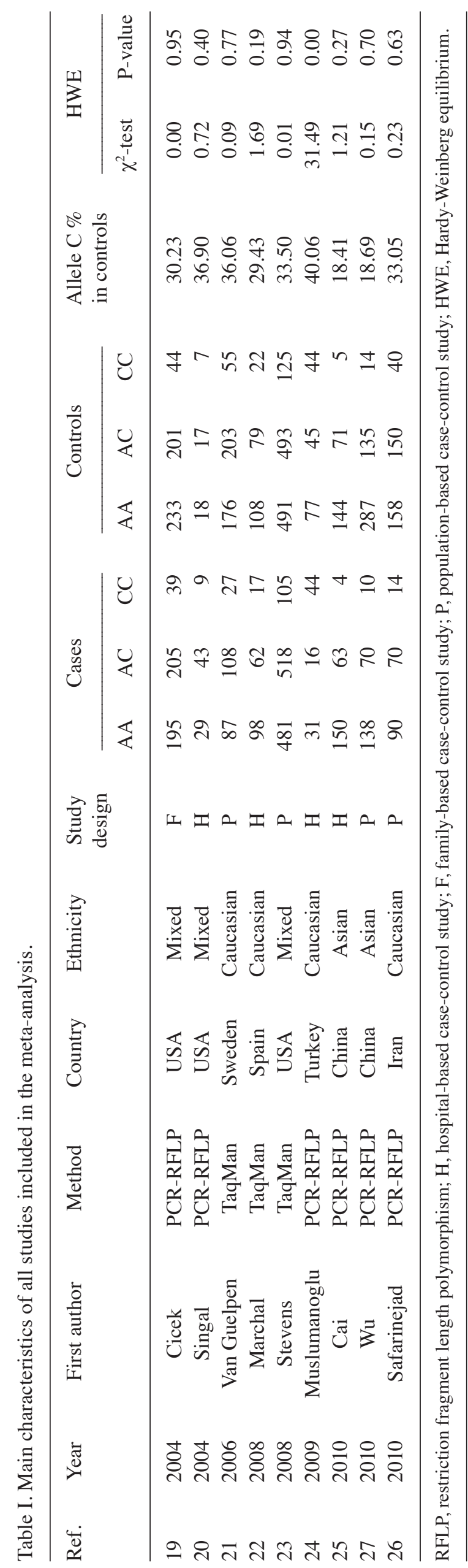




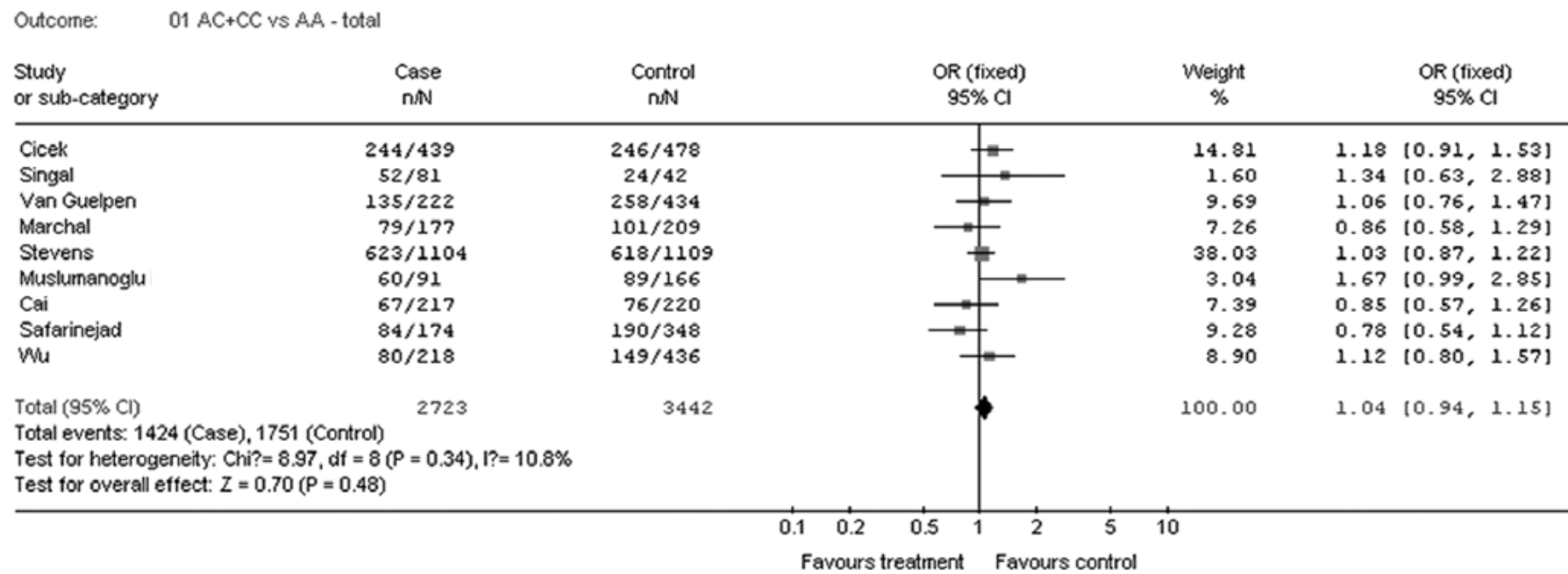

Figure 1. Association of the MTHFR gene A1298C polymorphism with the risk of prostate cancer in the dominant model: AC + CC vs. AA.

gene A1298C polymorphism and PC susceptibility in the electronic PubMed database (19-27). All of the qualified articles were case-control designed studies, consisting of a total of 2,723 PC cases and 3,442 controls. The detailed characteristics of the studies, such as year of publication, name of first author, country of origin, ethnicity, source of control groups, genotyping methods, $\mathrm{C}$ allele percentage in controls, HWE and the genotype distribution of the MTHFR gene A1298C polymorphism, are documented in Table I. Among the nine studies, two involved Asian populations $(25,27)$, four involved Caucasian populations $(21,22,24,26)$, and the remaining three were of mixed ethnicities $(19,20,23)$. All studies, but one (24), were consistent with HWE. As to the source of control groups, four were population-based studies $(21,23,26,27)$, four were hospital-based studies $(20,22,24,25)$ and the other one (19) was a family-based study. Only four studies provided data on disease stage, including advanced and localized PC $(19,20,22,26)$. PCR-RFLP was used to distinguish genotype in six studies (19,20,24-27), and TaqMan SNP genotyping assay was chosen for the other three (21-23). All of the research studies made use of DNA samples extracted from peripheral blood cells for genotyping, except one, which employed fixed tissue samples (20). Thus, subgroup analysis was conducted in this meta-analysis.

Test of heterogeneity and main results of the meta-analysis. Significant heterogeneity between studies $(\mathrm{P}<0.10)$ was observed in several comparisons, and the data are listed in Table II. The random-effects model (R) was chosen in the analysis when the P-value for the heterogeneity test was $<0.10$, otherwise the fixed-effects model $(\mathrm{F})$ was applied.

The results of the association between the MTHFR gene A1298C polymorphism and PC risk are also shown in Table II. Overall, when all the qualified studies were pooled into the meta-analysis, no evidence of significant association was found between PC risk and MTHFR gene 1298A>C polymorphism in any genetic model (codominant models: CC vs. AA, $\mathrm{OR}=1.03,95 \%$ CI $0.79-1.34, \mathrm{P}=0.84$; AC vs. $\mathrm{AA}, \mathrm{OR}=1.04$, 95\% CI 0.93-1.16, $\mathrm{P}=0.46$; dominant model: $\mathrm{AC}+\mathrm{CC}$ vs. AA, $\mathrm{OR}=1.04,95 \%$ CI 0.94-1.15, $\mathrm{P}=0.48$; recessive model: $\mathrm{CC}$ vs.
$\mathrm{AC}+\mathrm{AA}, \mathrm{OR}=1.02,95 \% \mathrm{CI}$ 0.76-1.35, $\mathrm{P}=0.91$; allele model: $\mathrm{C}$ vs. $\mathrm{A}, \mathrm{OR}=1.04,95 \%$ CI 0.90-1.19, $\mathrm{P}=0.61$ ) (Fig. 1).

In the stratified analyses by ethnicity, no significant results were found for Asian and Caucasian subjects in the different statistical models (all P>0.05). Moreover, meta-analyses of studies illustrating advanced and localized PC were conducted, and these analyses again found no significant correlations in any type of statistical model (all $\mathrm{P}>0.05$ ). Furthermore, insignificant statistical conclusions were found for hospital- and population-based subjects in various statistical models in the subgroup analyses according to source of controls (all $\mathrm{P}>0.05$ ).

Sensitivity analyses. In the study by Muslumanoglu et al (24), genotype frenquencies of the MTHFR gene A1298C polymorphism in the control group deviated from $\mathrm{HWE}(\mathrm{P}=0.00)$. Sensitivity analyses were carried out by excluding the above study and no evident changes were found for the pooled ORs. Similarly, the pooled ORs were not qualitatively influenced after exclusion of one heavily weighted study by Stevens et al (23). Sensitivity analyses suggested that the results of this meta-analysis were stable.

Publication bias. The shape of the funnel plots did not show any evidence of obvious asymmetry for all genetic models in either overall or stratified meta-analyses. Subsequently, Begger's funnel plot, Begger's test and Egger's test were performed to assess the publication bias of the eligible studies. Still, the results did not reveal obvious evidence of publication bias ( $\mathrm{P}=0.767$ for Egger's test in the dominant model; Fig. 2).

\section{Discussion}

MTHFR is involved in the one-carbon cycle, which is of importance for nucleotide synthesis and methylation of DNA, membranes, proteins and lipids. The MTHFR gene A1298C polymorphism, one of the most popular sites, is associated with a $30 \%$ decreased enzymatic activity without thermolability $(28,29)$. Thus, the MTHFR gene A1298C polymorphism is considered to produce the potentially functional site rs1801131, which was extensively studied. 
Table II. Main results of the meta-analysis in codominant, dominant, recessive and alleles models.

\begin{tabular}{|c|c|c|c|c|c|c|c|c|}
\hline Genetic models & No. of studies & $\mathrm{PC}(\mathrm{n})$ & OR & $95 \% \mathrm{CI}$ & $\mathrm{I}^{2}(\%)$ & $\mathrm{P}_{\mathrm{h}}$ & Statistical model & P-value \\
\hline \multicolumn{9}{|c|}{ Codominant models } \\
\hline \multicolumn{9}{|c|}{ AC vs. AA } \\
\hline Total & 9 & 2,454 & 1.04 & $0.93-1.16$ & 0 & 0.67 & $\mathrm{~F}$ & 0.46 \\
\hline PBC DNA & 8 & 2,382 & 1.03 & $0.93-1.15$ & 0 & 0.68 & $\mathrm{~F}$ & 0.55 \\
\hline Caucasian & 5 & 612 & 0.94 & $0.77-1.16$ & 0 & 0.68 & $\mathrm{~F}$ & 0.59 \\
\hline Asian & 2 & 421 & 0.97 & $0.75-1.27$ & 0 & 0.39 & $\mathrm{~F}$ & 0.85 \\
\hline H-based & 4 & 492 & 0.92 & $0.71-1.19$ & 0 & 0.60 & $\mathrm{~F}$ & 0.51 \\
\hline P-based & 4 & 1,562 & 1.04 & $0.91-1.19$ & 0 & 0.61 & $\mathrm{~F}$ & 0.58 \\
\hline HWE & 8 & 2,407 & 1.05 & $0.94-1.17$ & 0 & 0.59 & $\mathrm{~F}$ & 0.42 \\
\hline Advanced PC & 4 & 395 & 1.12 & $0.87-1.44$ & 39.9 & 0.17 & $\mathrm{~F}$ & 0.40 \\
\hline Localized PC & 4 & 355 & 0.95 & $0.73-1.24$ & 0 & 0.67 & $\mathrm{~F}$ & 0.69 \\
\hline \multicolumn{9}{|l|}{ CC vs. AA } \\
\hline Total & 9 & 1,568 & 1.03 & $0.79-1.34$ & 41.7 & 0.09 & $\mathrm{R}$ & 0.84 \\
\hline PBC DNA & 8 & 1,530 & 1.04 & $0.78-1.39$ & 48.4 & 0.06 & $\mathrm{R}$ & 0.78 \\
\hline Caucasian & 5 & 432 & 1.01 & $0.59-1.73$ & 66.4 & 0.02 & $\mathrm{R}$ & 0.98 \\
\hline Asian & 2 & 302 & 1.22 & $0.60-2.49$ & 0 & 0.41 & $\mathrm{~F}$ & 0.58 \\
\hline H-based & 4 & 382 & 1.18 & $0.60-2.33$ & 58.9 & 0.06 & $\mathrm{R}$ & 0.63 \\
\hline P-based & 4 & 952 & 0.88 & $0.70-1.10$ & 0 & 0.41 & F & 0.26 \\
\hline HWE & 8 & 1,493 & 0.90 & 0.74-1.09 & 0 & 0.84 & $\mathrm{~F}$ & 0.27 \\
\hline Advanced PC & 4 & 233 & 0.78 & $0.50-1.20$ & 44.5 & 0.14 & $\mathrm{R}$ & 0.26 \\
\hline Localized PC & 4 & 242 & 1.03 & $0.68-1.54$ & 0 & 0.89 & F & 0.90 \\
\hline \multicolumn{9}{|l|}{ Dominant model } \\
\hline \multicolumn{9}{|l|}{$\mathrm{CC}+\mathrm{AC}$ vs. AA } \\
\hline Total & 9 & 2,723 & 1.04 & $0.94-1.15$ & 10.8 & 0.34 & $\mathrm{~F}$ & 0.48 \\
\hline PBC DNA & 8 & 2,642 & 1.03 & $0.93-1.15$ & 19.0 & 0.28 & $\mathrm{~F}$ & 0.53 \\
\hline Caucasian & 5 & 720 & 0.99 & $0.82-1.20$ & 36.7 & 0.18 & $\mathrm{~F}$ & 0.94 \\
\hline Asian & 2 & 435 & 0.99 & $0.77-1.29$ & 6.8 & 0.30 & $\mathrm{~F}$ & 0.96 \\
\hline H-based & 4 & 566 & 1.02 & $0.81-1.30$ & 44.2 & 0.15 & $\mathrm{~F}$ & 0.84 \\
\hline P-based & 4 & 1,718 & 1.01 & $0.89-1.15$ & 0 & 0.48 & $\mathrm{~F}$ & 0.88 \\
\hline HWE & 8 & 2,632 & 1.02 & $0.92-1.13$ & 0 & 0.56 & $\mathrm{~F}$ & 0.72 \\
\hline Advanced PC & 4 & 431 & 1.04 & $0.82-1.33$ & 49.0 & 0.12 & $\mathrm{~F}$ & 0.74 \\
\hline Localized PC & 4 & 400 & 0.97 & $0.75-1.24$ & 0 & 0.86 & F & 0.79 \\
\hline \multicolumn{9}{|l|}{ Recessive model } \\
\hline \multicolumn{9}{|l|}{$\mathrm{CC}$ vs. $\mathrm{AA}+\mathrm{AC}$} \\
\hline Total & 9 & 2,723 & 1.02 & $0.76-1.35$ & 53.4 & 0.03 & $\mathrm{R}$ & 0.91 \\
\hline PBC DNA & 8 & 2,642 & 1.05 & $0.77-1.42$ & 57.5 & 0.02 & $\mathrm{R}$ & 0.77 \\
\hline Caucasian & 5 & 720 & 1.00 & $0.57-1.76$ & 73.5 & 0.01 & $\mathrm{R}$ & 0.99 \\
\hline Asian & 2 & 435 & 1.22 & $0.60-2.47$ & 0 & 0.46 & F & 0.58 \\
\hline H-based & 4 & 566 & 1.15 & $0.55-2.41$ & 68.7 & 0.02 & $\mathrm{R}$ & 0.71 \\
\hline P-based & 4 & 1,718 & 0.86 & $0.69-1.07$ & 0 & 0.51 & F & 0.17 \\
\hline HWE & 8 & 2,632 & 0.87 & $0.73-1.04$ & 0 & 0.89 & $\mathrm{~F}$ & 0.14 \\
\hline Advanced PC & 4 & 431 & 0.71 & $0.47-1.09$ & 32.4 & 0.22 & $\mathrm{~F}$ & 0.12 \\
\hline Localized PC & 4 & 400 & 1.05 & $0.71-1.56$ & 0 & 0.74 & F & 0.80 \\
\hline \multicolumn{9}{|l|}{ Allele model } \\
\hline \multicolumn{9}{|l|}{ C vs. A } \\
\hline Total & 9 & 5,446 & 1.04 & $0.90-1.19$ & 57.7 & 0.02 & $\mathrm{R}$ & 0.61 \\
\hline PBC DNA & 8 & 5,284 & 1.04 & $0.90-1.20$ & 63.0 & 0.01 & $\mathrm{R}$ & 0.62 \\
\hline Caucasian & 5 & 1,440 & 1.05 & $0.77-1.43$ & 76.2 & 0.00 & $\mathrm{R}$ & 0.76 \\
\hline Asian & 2 & 870 & 1.02 & $0.81-1.27$ & 24.6 & 0.25 & F & 0.89 \\
\hline H-based & 4 & 1,132 & 1.12 & $0.75-1.69$ & 78.0 & 0.00 & $\mathrm{R}$ & 0.58 \\
\hline P-based & 4 & 3,436 & 0.98 & $0.89-1.08$ & 8.9 & 0.35 & F & 0.62 \\
\hline HWE & 8 & 5,264 & 0.98 & $0.91-1.07$ & 0 & 0.62 & $\mathrm{~F}$ & 0.69 \\
\hline Advanced PC & 4 & 862 & 0.96 & $0.80-1.15$ & 50.8 & 0.11 & $\mathrm{~F}$ & 0.65 \\
\hline Localized PC & 4 & 800 & 1.01 & $0.84-1.23$ & 0 & 0.97 & $\mathrm{~F}$ & 0.88 \\
\hline
\end{tabular}

PC, prostate cancer; $\mathrm{P}_{\mathrm{h}}$, P-value of Q test for heterogeneity test; H-based, hospital-based case-control study; P, population-based case-control study; F, fixed-effects model; R, random-effects model; HWE, Hardy-Weinberg equilibrium; PBC, peripheral blood cell. 


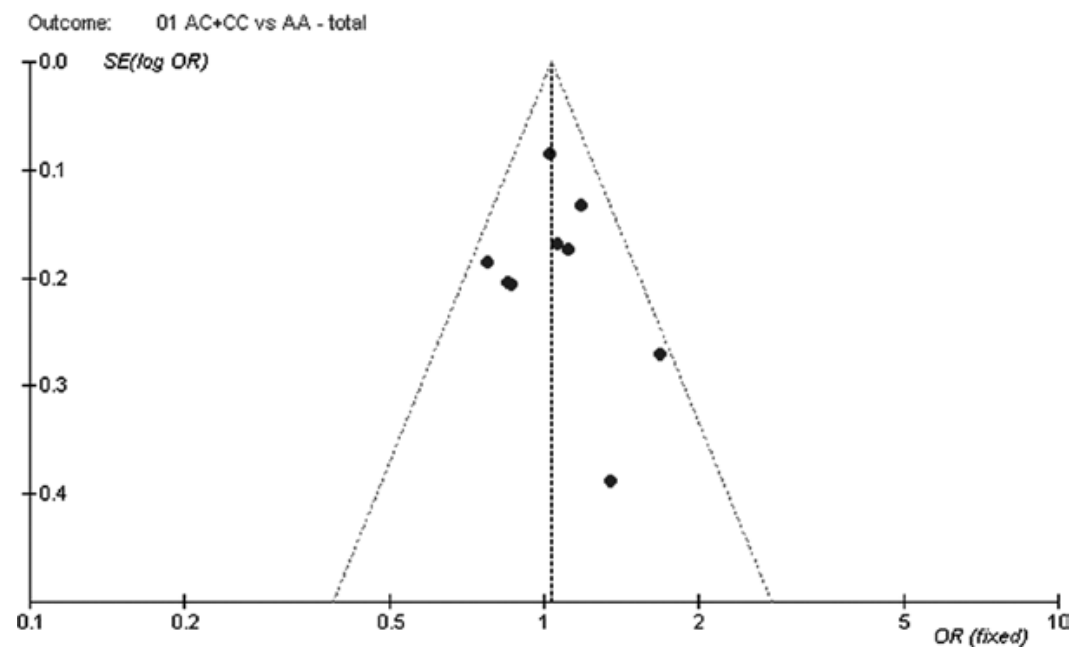

Figure 2. Funnel plot for the relationship of the MTHFR gene A1298C polymorphism with the risk of prostate cancer in the dominant model: AC + CC vs. AA.

The published literature regarding the association between the MTHFR gene A1298C polymorphism and the risk of PC consists of small studies mainly in Caucasian populations, which show conflicting findings. No consensus has yet been reached. To date, there have been two meta-analyses $(30,31)$ focusing on the association between the two, both of which found that the MTHFR gene A1298C polymorphism did not contribute to the susceptibility of PC. However, the two previous meta-analyses included only a small number of casecontrol studies, with limited Caucasian subjects. Therefore, we performed a meta-analysis of all eligible studies, in order to derive a more conclusive estimation of the relationship. A total of nine case-control studies were selected in our meta-analysis, consisting of 2,723 PC cases and 3,442 controls. In the total population, we did not find an association between the MTHFR gene A1298C polymorphism and PC risk in the codominant, dominant, recessive and allele models (all $\mathrm{P}>0.05$ ).

In the eligible studies, the percentage of the $\mathrm{C}$ allele was 0.1841 (25) and 0.1869 (27) in Asian population, while it was $>0.25 \%$ for other populations. Hence, the different genetic background may have affected the results of the meta-analysis. In the stratified analysis by ethnicity, no significant association between the MTHFR gene A1298C polymorphism and PC risk was found for either Caucasian or Asian populations in the codominant, dominant, recessive and allele models (all $\mathrm{P}>0.05)$. Furthermore, no statistically significant results were found between the MTHFR gene A1298C polymorphism and PC development in the subgroup analyses of HWE, PBC DNA, advanced PC, localized PC, hospital-based and populationbased case control studies (all $\mathrm{P}>0.05$ ).

The results of the present study, along with those of other meta-analyses regarding the MTHFR gene A1298C polymorphism (32-34), reached the same conclusions, which found no association between the polymorphism site and the disease. It may not be uncommon that the results of the funciional study were not coincident with the epidemiological results. The mentioned discrepancy may be due to complex genetic background and multi-genetic interaction (35). On the other hand, one recent study highlighted that the MTHFR gene A1298C polymorphism is not associated with the modification of MTHFR activity (36). As a result, the specific mechanism of how the MTHFR gene A1298C polymorphism influences the MTHFR function warrants further investigation.

In conclusion, this meta-analysis suggests that the MTHFR gene A1298C polymorphism is not associated with prostate cancer susceptibility in either total or stratified populations. Furthermore, gene-gene and gene-environment interactions should also be considered in the analysis, which may contribute to a better understanding of the possible genetic risk of prostate carcinoma.

\section{References}

1. Jemal A, Siegel R, Xu J and Ward E: Cancer statistics, 2010. CA Cancer J Clin 60: 277-300, 2010.

2. Bray F, Sankila R, Ferlay J and Parkin DM: Estimates of cancer incidence and mortality in Europe in 1995. Eur J Cancer 38: 99-166, 2002.

3. Hsing AW and Chokkalingam AP: Prostate cancer epidemiology. Front Biosci 11: 1388-1413, 2006.

4. Jung KW, Won YJ, Park S, et al: Cancer statistics in Korea: incidence, mortality and survival in 2005. J Korean Med Sci 24: 995-1003, 2009.

5. Mason JB and Levesque T: Folate: effects on carcinogenesis and the potential for cancer chemoprevention. Oncology 10: 1727-1736, 1742-1724, 1996.

6. Kim YI: Folate and carcinogenesis: evidence, mechanisms, and implications. J Nutr Biochem 10: 66-88, 1999.

7. Goyette P, Sumner JS, Milos R, et al: Human methylenetetrahydrofolate reductase: isolation of cDNA, mapping and mutation identification. Nat Genet 7: 195-200, 1994.

8. Frosst P, Blom HJ, Milos R, et al: A candidate genetic risk factor for vascular disease: a common mutation in methylenetetrahydrofolate reductase. Nat Genet 10: 111-113, 1995.

9. Dobosy JR, Roberts JL, Fu VX and Jarrard DF: The expanding role of epigenetics in the development, diagnosis and treatment of prostate cancer and benign prostatic hyperplasia. J Urol 177: 822-831, 2007.

10. Murphy TM, Perry AS and Lawler M: The emergence of DNA methylation as a key modulator of aberrant cell death in prostate cancer. Endocr Relat Cancer 15: 11-25, 2008.

11. Goyette P, Pai A, Milos R, et al: Gene structure of human and mouse methylenetetrahydrofolate reductase (MTHFR). Mamm Genome 9: 652-656, 1998.

12. Kurzawski M, Dziewanowski K, Kędzierska K, Wajda A, Lapczuk J and Droździk M: Association of transcription factor 7-like 2 (TCF7L2) gene polymorphism with posttransplant diabetes mellitus in kidney transplant patients medicated with tacrolimus. Pharmacol Rep 63: 826-833, 2011. 
13. Leu JD, Wang CY, Tsai HY, Lin IF, Chen RC and Lee YJ: Involvement of p53 R72P polymorphism in the association of MDM2-SNP309 with breast cancer. Oncol Rep 25: 1755-1763, 2011.

14. Kim JW, Park HM, Choi YK, Chong SY, Oh D and Kim NK: Polymorphisms in genes involved in folate metabolism and plasma DNA methylation in colorectal cancer patients. Oncol Rep 25: 167-172, 2011.

15. Fung MM, Salem RM, Lipkowitz MS, et al: Methylenetetrahydrofolate reductase (MTHFR) polymorphism A1298C (Glu429Ala) predicts decline in renal function over time in the African-American Study of Kidney Disease and Hypertension (AASK) Trial and Veterans Affairs Hypertension Cohort (VAHC). Nephrol Dial Transplant: May, 2011 (E-pub ahead of print).

16. Dayakar S, Goud KI, Reddy TP, Rao SP, Sesikeran SB and Sadhnani M: Sequence variation of the methylene tetrahydrofolate reductase gene $(677 \mathrm{C}>\mathrm{T}$ and $1298 \mathrm{~A}>\mathrm{C})$ and traditional risk factors in a South Indian population. Genet Test Mol Biomarkers: July, 2011 (E-pub ahead of print).

17. Siemianowicz K, Gminski J, Garczorz W, et al: Methylenetetrahydrofolate reductase gene C677T and A1298C polymorphisms in patients with small cell and non-small cell lung cancer. Oncol Rep 10: 1341-1344, 2003.

18. Shen H, Newmann AS, Hu Z, et al: Methylenetetrahydrofolate reductase polymorphisms/haplotypes and risk of gastric cancer: a case-control analysis in China. Oncol Rep 13: 355-360, 2005.

19. Cicek MS, Nock NL, Li L, Conti DV, Casey G and Witte JS Relationship between methylenetetrahydrofolate reductase C677T and A1298C genotypes and haplotypes and prostate cancer risk and aggressiveness. Cancer Epidemiol Biomarkers Prev 13: 1331-1336, 2004.

20. Singal R, Ferdinand L, Das PM, Reis IM and Schlesselman JJ: Polymorphisms in the methylenetetrahydrofolate reductase gene and prostate cancer risk. Int J Oncol 25: 1465-1471, 2004.

21. Van Guelpen BR, Wirén SM, Bergh AR, Hallmans G, Stattin PE and Hultdin J: Polymorphisms of methylenetetrahydrofolate reductase and the risk of prostate cancer: a nested case-control study. Eur J Cancer Prev 15: 46-50, 2006.

22. Marchal C, Redondo M, Reyes-Engel A, et al: Association between polymorphisms of folate-metabolizing enzymes and risk of prostate cancer. Eur J Surg Oncol 34: 805-810, 2008.

23. Stevens VL, Rodriguez C, Sun J, Talbot JT, Thun MJ and Calle EE: No association of single nucleotide polymorphisms in one-carbon metabolism genes with prostate cancer risk. Cancer Epidemiol Biomarkers Prev 17: 3612-3614, 2008.

24. Muslumanoglu MH, Tepeli E, Demir S, et al: The analysis of the relationship between A1298C and C677T polymorphisms of the MTHFR gene with prostate cancer in Eskisehir population. Genet Test Mol Biomarkers 13: 641-645, 2009.
25. Cai D, Ning L, Pan C, et al: Association of polymorphisms in folate metabolic genes and prostate cancer risk: a case-control study in a Chinese population. J Genet 89: 263-267, 2010.

26. Safarinejad MR, Shafiei N and Safarinejad S: Relationship between three polymorphisms of methylenetetrahydrofolate reductase (MTHFR C677T, A1298C, and G1793A) gene and risk of prostate cancer: a case-control study. Prostate 70: 1645-1657, 2010.

27. Wu HC, Chang CH, Tsai RY, et al: Significant association of methylenetetrahydrofolate reductase single nucleotide polymorphisms with prostate cancer susceptibility in Taiwan. Anticancer Res 30: 3573-3577, 2010.

28. Weisberg IS, Jacques PF, Selhub J, et al: The 1298A-->C polymorphism in methylenetetrahydrofolate reductase (MTHFR): in vitro expression and association with homocysteine. Atherosclerosis 156: 409-415, 2001.

29. van der Put NM, Gabreëls F, Stevens EM, et al: A second common mutation in the methylenetetrahydrofolate reductase gene: an additional risk factor for neural-tube defects? Am J Hum Genet 62: 1044-1051, 1998.

30. Bai JL, Zheng MH, Xia X, Ter-Minassian M, Chen YP and Chen F: MTHFR C677T polymorphism contributes to prostate cancer risk among Caucasians: a meta-analysis of 3511 cases and 2762 controls. Eur J Cancer 45: 1443-1449, 2009.

31. Collin SM, Metcalfe C, Zuccolo L, et al: Association of folatepathway gene polymorphisms with the risk of prostate cancer: a population-based nested case-control study, systematic review, and meta-analysis. Cancer Epidemiol Biomarkers Prev 18: 2528-2539, 2009

32. Dong X, Wu J, Liang P, Li J, Yuan L and Liu X: Methylenetetrahydrofolate reductase C677T and A1298C polymorphisms and gastric cancer: a meta-analysis. Arch Med Res 41: 125-133, 2010.

33. Qiu LX, Zhang J, Li WH, et al: Lack of association between methylenetetrahydrofolate reductase gene A1298C polymorphism and breast cancer susceptibility. Mol Biol Rep 38: 2295-2299, 2011.

34. Wei B, Xu Z, Ruan J, et al: MTHFR 677C>T and 1298A>C polymorphisms and male infertility risk: a meta-analysis. Mol Biol Rep: June, 2011 (E-pub ahead of print).

35. Hirschhorn JN, Lohmueller K, Byrne E and Hirschhorn K: A comprehensive review of genetic association studies. Genet Med 4: 45-61, 2002.

36. Lievers KJ, Boers GH, Verhoef $\mathrm{P}$, et al: A second common variant in the methylenetetrahydrofolate reductase (MTHFR) gene and its relationship to MTHFR enzyme activity, homocysteine, and cardiovascular disease risk. J Mol Med (Berl) 79: 522-528, 2001. 